\title{
Region specificity of rectus femoris muscle for force vectors in vivo.
}

\section{$\operatorname{AUTHOR}(\mathrm{S})$ :}

Hagio, Shota; Nagata, Kaori; Kouzaki, Motoki

\section{CITATION:}

Hagio, Shota ...[et al]. Region specificity of rectus femoris muscle for force vectors in vivo.. Journal of biomechanics 2012, 45(1): 179-182

\section{ISSUE DATE:}

2012-01-03

URL:

http://hdl.handle.net/2433/152366

\section{RIGHT:}

(C) 2011 Elsevier Ltd.; This is not the published version. Please cite only the published version.; この論文は出版社版でありません。引用の際に は出版社版をご確認ご利用ください。 


\title{
Region specificity of rectus femoris muscle for force vectors in vivo
}

\author{
Shota Hagio*, Kaori Nagata*, and Motoki Kouzaki* \\ * Laboratory of Neurophysiology, Graduate School of Human and Environmental Studies, \\ Kyoto University, Sakyo-ku, Kyoto, Japan.
}

Keywords: bi-articular muscle, wire electrode, electrical stimulation

Author for correspondence:

Motoki Kouzaki, PhD

Laboratory of Neurophysiology

Graduate School of Human and Environmental Studies

Kyoto University

Yoshida-nihonmatsu-cho, Sakyo-ku, Kyoto, Japan 606-8501

Tel \& Fax: +81-75-753-2927

E-mail: m.kouzaki@kt8.ecs.kyoto-u.ac.jp 


\begin{abstract}
To examine the region specificity within the rectus femoris (RF) for knee extension and hip flexion force directions, three force components around the ankle were measured during intramuscular electrical stimulation applied to six parts of the RF: a proximal and medial part, a proximal and lateral part, a middle and medial part, a middle and lateral part, a distal and medial part, and a distal and lateral part. As a result, the exerted force directions in all of the subjects were variable in all regions, and the proximal region of the RF was the dominant contributor to the hip flexion moment. In addition, the force in the lateral region of the $\mathrm{RF}$, rather than that in the medial region, denoted the lateral direction. These results suggest that divergent regions of muscle fibers within the RF are responsible for different functions in determining the force direction.
\end{abstract}




\section{Introduction}

It has been proposed that the bi-articular muscles contribute to controlling the direction of the limb endpoint (Jacobs and van Ingen Schenau, 1992; Van Bolhuis et al., 1998), and that the mono-articular muscles have a role for generating net moment. According to the both biomechanical and physiological literature, it has been considered that the activities for controlling multi-joint movements of bi-articular muscles are more important than mono-articular muscles for smooth human movements. In addition, the anatomical features of the bi-articular muscles make it more difficult to understand the redundant and complex activities, because they generate interaction between adjacent joint torques. For example, the activity of the rectus femoris (RF) muscle produces knee extension as well as hip flexion torques because it spans both the knee and hip joints. The problem to consider here is how the RF simultaneously regulates the knee extension and hip flexion torques. According to human cadaver studies, the proximal and distal regions of RF receive different innervations by two large branches originating from the femoral nerve (Daping and Steven, 1999; Sung et al., 2003). This evidence led us to hypothesize that divergent regions of muscle fibers within RF have different functions for determining the net joint moments. The purpose of the present study was to verify our hypothesis and to further understand the functions of RF muscle as bi-articular muscles.

\section{Methods}

\subsection{Subjects}

Seven healthy male subjects voluntarily participated in this study. The age, height, and body mass of the subjects (mean $\pm \mathrm{SD}$ ) were $27.2 \pm 6.2 \mathrm{yr}, 171.2 \pm 6.5 \mathrm{~cm}$, and $65.5 \pm 5.5$ $\mathrm{kg}$, respectively. They gave their written informed consent to participate in the study after receiving a detailed explanation of the purposes, potential benefits, and risks associated with participation. All procedures used in this study were in accordance with the 
Declaration of Helsinki and were approved by the Committee for Human Experimentation at the Graduate School of Human and Environmental Studies, Kyoto University.

\subsection{Measurements and procedures}

The subjects were seated comfortably in a chair with the hip and knee flexed at $90^{\circ}$ from full extension. The upper body was strapped to the chair so that the subjects maintained a stable position throughout the experiment. The three-vector isometric forces were measured by a tri-axial force transducer (LSM-B-500NSA1, Kyowa, Tokyo, Japan). The tri-axial force transducer was attached to the right ankle, and three force vectors in the upward-downward $(F x)$, medial-lateral $(F y)$, and anterior-posterior $(F z)$ directions around the ankle joint (Kouzaki et al., 2002) were measured (Fig. 1A).

A pair of custom-made intramuscular wire electrodes was used to stimulate the six regions of RF. The intramuscular electrodes were made by threading a stainless-steel wire $(\varphi=50 \mu \mathrm{m})$ with a Teflon coating through a 25 -gauge hypodermic needle, stripping the Teflon coating off the wire tip ( $\sim 2 \mathrm{~mm})$, and bending the tip over the needle point to form a hook. To exclude cross-talk, short inter-electrode distance $(<2 \mathrm{~mm})$ and small electrode diameter for electrical stimulation were applied. The needle was inserted into the six regions (determined in the subsequent sentence) of RF and then gently removed, leaving the wire electrode in place. The electrode was implanted into the RF muscle to a depth of $\sim 25 \mathrm{~mm}$. Tetanic electrical stimulation was used to activate each of the six regions of RF in the right leg. Stimulation intensity was supramaximal. The tetanic stimulation consisted of a $1 \mathrm{~ms}$ rectangular pulse at $50 \mathrm{~Hz}$ for $5 \mathrm{~s}$.

To determine the insertion sites of intramuscular wire electrodes as well as the shape, intramuscular aponeurosis, and fascicles of RF, cross-sectional images from the proximal to distal regions of the RF were obtained with a B-mode ultrasonic apparatus (SSD-900, 
Aloka, Tokyo, Japan). The six regions for insertion were a proximal and medial part (PM), a proximal and lateral part (PL), a middle and medial part (MM), a middle and lateral part (ML), a distal and medial part (DM), and a distal and lateral part (DL) (Fig. 1B). These parts were determined on the basis of the morphological characteristics of the RF muscle. The linear distances between every part and the superior border of the patella were measured. For the proximal parts, they were $70 \pm 3 \%$ of the total length between the upper patella and anterior inferior iliac spine; the middle parts, $60 \%$; the distal parts, $35 \%$. Here, we carefully describe that only the proximal region was provided the standard deviation because of the different architecture on this region in every subject. The medial and lateral parts in the middle and distal parts were separated by the intramuscular aponeurosis of RF.

< Insert Figure 1 about here >

Each of the six regions of RF was electrically stimulated. Between stimulations, sufficient rest of at least 5 min was taken. The order of the stimulations was randomized. During electrical stimulations, each subject remained in a relaxed state. The force signals measured by the tri-axial force transducer were stored with a sample frequency of 1,000 $\mathrm{Hz}$ on the hard disk of a personal computer using a 16-bit analog-to-digital converter (PowerLab/16SP; ADInstruments, Sydney, Australia).

\subsection{Data analysis}

The force signals measured by the tri-axial force transducer were digitized and analyzed in every stimulation task. To determine the three-dimensional direction of the force exerted by electrically stimulating every part of the RF, the steady periods of three forces $(F x, F y$, and $F z)$ during electrical stimulation for approximately $1 \mathrm{~s}$ were extracted 
for later analyses, and the mean values of the respective forces were calculated. Then, from the mean values, a point $(\boldsymbol{r})$ in spatial coordinates (x-axis: $F y$, y-axis: $F z$, z-axis: $F x$ ) was calculated by using the following equation.

$$
\boldsymbol{r}=\sqrt{F x^{2}+F y^{2}+\mathrm{Fz}^{2}}
$$

Moreover, in the ankle, the point that the x-axis, $y$-axis and z-axis of tri-axial force transducer were intersected was defined as the "origin". Based on the above calculation, a vector from the origin to the point $(\boldsymbol{r})$ was defined as the three-dimensional force direction.

\section{Results}

The calculated force directions in the six regions are shown in Fig. 2. Variability of the force directions in every part was represented as the standard deviation of the end points of the vectors (PL: 0.431, PM: 0.285, ML: 0.276, MM: 0.205, DL: 0.310, DM: 0.348). The force directions in every subject were variable in all regions. For the proximal region, PL and PM had a relatively upward direction in comparison with the middle and distal regions. In almost all of the subjects, the force denoted the lateral direction. Especially in the lateral regions, i.e., PL, ML, and DL, the forces were lateral-directed rather than in the medial regions.

< Insert Figure 2 about here >

\section{Discussion}

\subsection{Upward direction of force in proximal region of $\mathbf{R F}$}

The proximal region, i.e., PL and PM, had an upward direction in comparison with the middle and distal regions, indicating that the proximal region dominantly contributed to exerting the hip flexion moment. The series elastic component of the RF consists of not 
only the anterior and posterior sites of aponeurosis but also intramuscular aponeurosis (Arai et al., 1993; Daping and Steven, 1999; Hasselman et al., 1995). The anterior aponeurosis extends over the proximal third of the RF, and the superior one over the distal two-thirds. The intramuscular aponeurosis is located on the longitudinal center line of the RF and spans the majority of the muscle length. Moreover, a tendon was shown to be much stiffer than an aponeurosis (Maganaris and Paul, 2000). Namely, the force transmission efficiency of a tendon is higher than that of an aponeurosis. From anatomical facts, it is considered that in the proximal region of RF the force transmission efficiency to the hip joint is relatively high, and in the distal region it is low. Hence, the proximal region dominantly contributes to exerting the hip flexion moment.

\subsection{Lateral direction of force}

In most of the subjects, the force in every part denoted the lateral direction. This result is considered to relate the anatomical feature of the knee joint; the knee extension leads the external tibial rotation (Moglo and Shirazi-Adl, 2005). Moreover, in the lateral region of the $\mathrm{RF}$, the forces were laterally directed relative to the case in the medial region. This is supported by the anatomical feature in which the muscle fibers of the lateral region were more exterior than in medial region and pull the distal tendon of RF (or the tendon of the quadriceps femoris muscle) more laterally. This signifies that divergent regions of muscle fibers within the RF have different functions for determining force direction.

\subsection{Anatomical features of RF}

Numerous anatomical complexities in the RF have been reported by many researchers (Balius et al., 2009; Hasselman et al., 1995). We obtained successive ultrasonic images from proximal to distal portions of RF as typical subject (Fig. 3). As a result, a lot of 
aponeuroses were complexly complicated within the RF. Such architectural feature of the RF might produce the heterogeneous force transmission. Therefore, the architecture of the RF cannot be represented by a simple rigid model, and it is thought that the results of the present study were related to these complicated architectures.

$<$ Insert Figure 3 about here>

\section{Conclusion}

In the present study we have found that: (1) the force directions in all of the subjects were variable in all regions, (2) the proximal region of RF dominantly contributed to the hip flexion moment, and (3) the force in the lateral region, rather than that in the medial region, was lateral-directed. These findings support our hypothesis that divergent regions of muscle fibers within RF have different functions for determining the force direction.

\section{Conflict of Interest Statement}

There is no conflict of interest.

\section{Acknowledgment}

We are most grateful to Professor Toshio Moritani of Kyoto University, giving us invaluable teachings on the study. This study was performed under medical supervision by Tatsuya Hayashi, M.D., Ph.D., Kyoto University. This work was supported, in part, by Grant from Tateisi Science and Technology Foundation (to M.K.). 


\section{References}

Arai T. et al. A study of arterial supply in the human rectus femoris muscle. Plastic and reconstructive surgery. 92, 43-48 (1993)

Balius R. et al. Central aponeurosis tears of the rectus femoris: practical sonographic prognosis. Br. J. Sports Med.43, 818-824 (2009)

Daping Y. \& Steven F. M. Neurovascular anatomy of the rectus femoris muscle related to functioning muscle transfer. Plastic and reconstructive surgery. 104, 102-106 (1999)

Hasselman C. T. et al. An explanation for various rectus femoris strain injuries using previously undescribed muscle architecture. The American Journal of Sports Medicine. 23, 493-499 (1995)

Jacobs R. \& van Ingen Schenau G. J. Control of an external force in leg extensions in humans. Journal of Phisiology. 457, 611-626 (1992).

Kouzaki M. et al. Alternate muscle activity observed between knee extensor synergists during low-level sustained contractions. Journal of Applied Physiology. 93, 675-684 (2002).

Maganaris CN \& Paul JP. Load-elongation characteristics of in vivo human tendon and aponeurosis. Journal of Experimental Biology. 203, 751-756 (2000)

Moglo K. E. \& Shirazi-Adl Cruciate coupling and screw-home mechanism in passive knee joint during extension-flexion. Journal of Biomechanics. 38, 1075-1083 (2005)

Sung D. H. et al. Motor branch of rectus femoris: anatomic location for selective motor branch in stiff-legged gait. Arch Phys Med Rehabil. 84, 1028-1031 (2003)

Van Bolhuis B. M. et al. Activation patterns of mono- and bi-articular arm muscles as a function of force and movement direction of the wrist in humans. Journal of 
Physiology. 508, 313-324 (1998)

Zhang L. Q. et al. In vivo load sharing among the quadriceps components. Journal of Orthopaedic Research. 21, 565-571 (2003)

\section{Figure legends}

Figure 1: A. Experimental setup for measurement of $F x, F y$, and $F z$ of the right leg. B. Illustration of a right anterior thigh and the six parts of the RF where the wire electrodes for the electrical stimulation were inserted. The six parts were a proximal and medial part $(\mathrm{PM})$, a proximal and lateral part (PL), a middle and medial part (MM), a middle and lateral part (ML), a distal and medial part (DM), and a distal and lateral part (DL) of RF. The linear distances between every part and the superior border of the patella were measured. See text for further explanation.

Figure 2: Force directions in every part. The vectors (thin lines) in every figure indicate the force directions of individual subjects, and the thick line represents the mean value. To more easily compare differences among force directions, each line was transformed into the same length.

Figure 3: Ultrasonic images of cross-sectional sections of rectus femoris (RF) in one subject. Successive images of the anterior aspect of thigh from proximal to distal portions every $2 \mathrm{~cm}$. Since the RF extends transversally with proximal sites, we took parallel 2 images in proximal thigh segment. * indicates insertion points for intramuscular wire electrodes. VL, vastus lateralis; VI, vastus intermedius. 
A

B
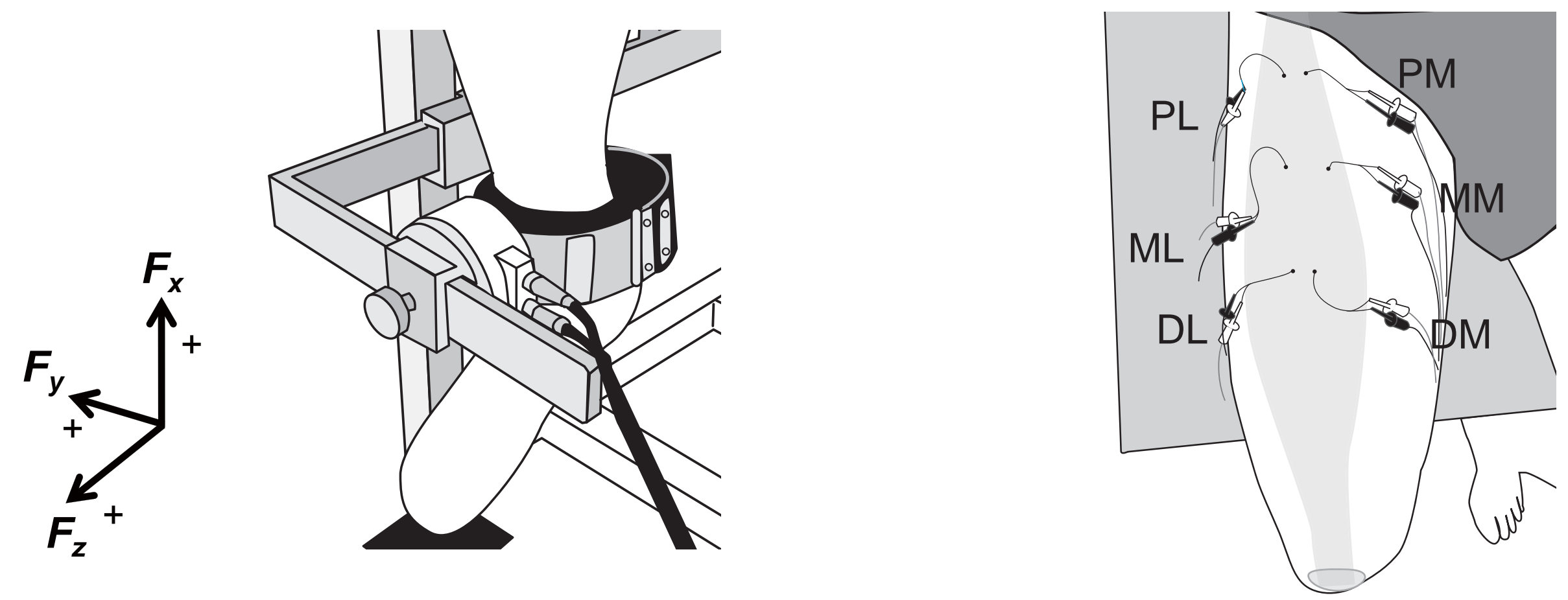

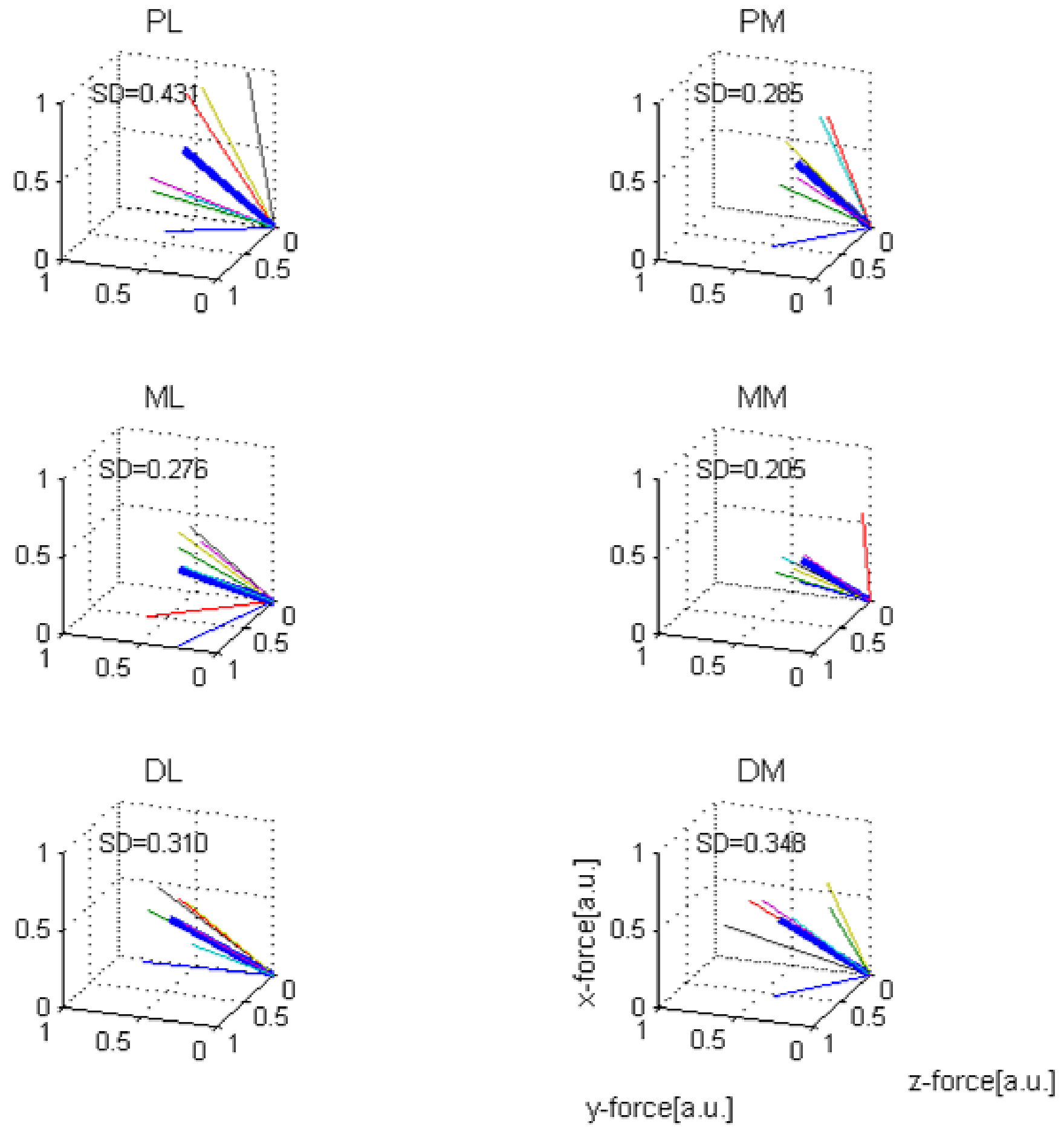


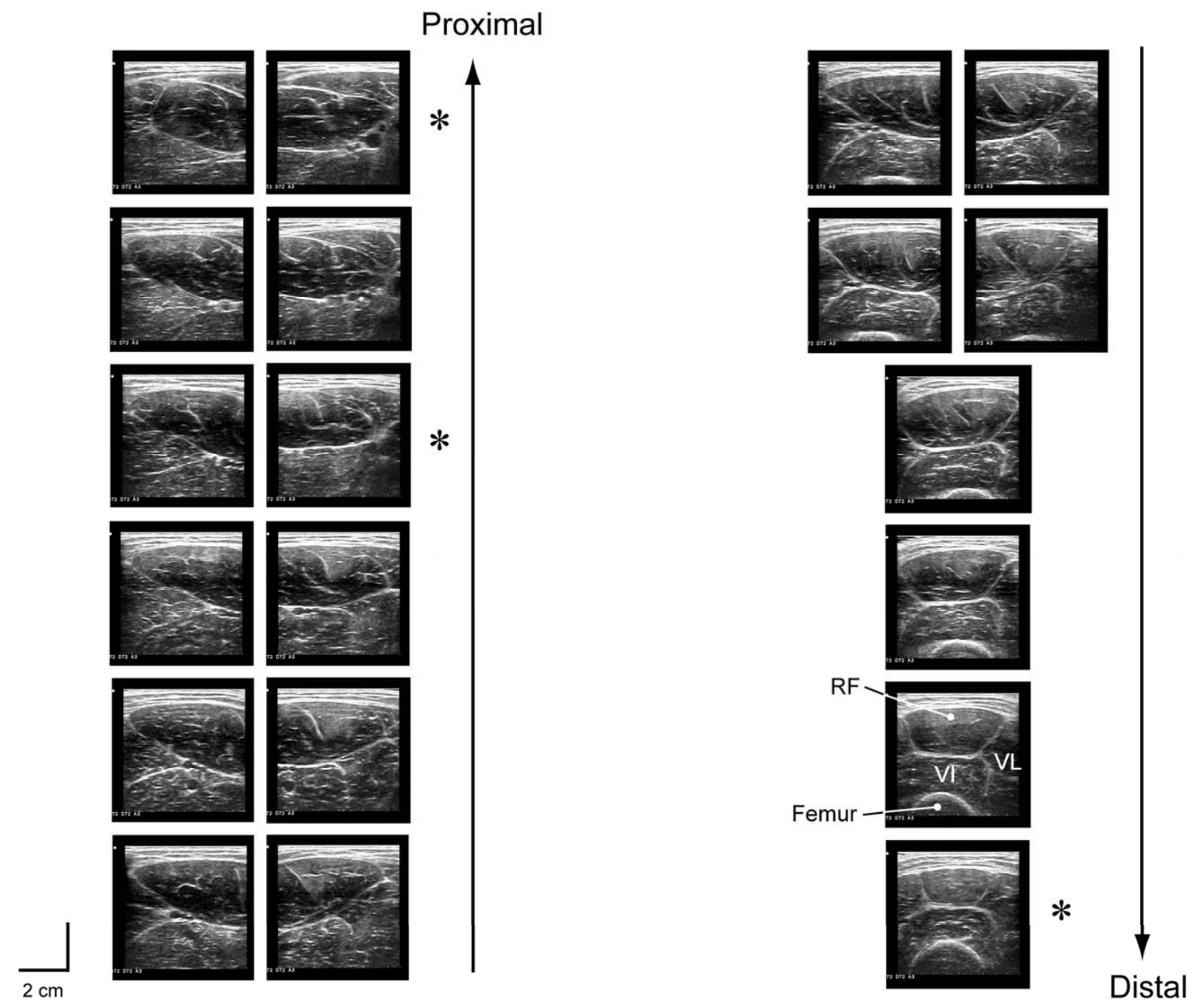

